Ann. Génét. Sél. anim., 1975, 7 (2), 197-204.

\title{
ÉTUDE DES LIAISONS ENTRE COMPORTEMENT EN OPEN-FIELD ET ÉMOTIVITÉ CHEZ LE JEUNE POUSSIN
}

\author{
J. M. FAURE \\ avec la collaboration technique de F. Morozeau \\ Station expérimentale d'Aviculture du Magneraud, I. N. R. A., \\ B. P. 52, 17700 Surgères (France)
}

\begin{abstract}
RÉSUMÉ
La liaison entre activité en open-field et émotivité a été étudiée dans deux souches de poussins sélectionnés pour leur activité en open-field à l'âge de deux jours. Quatre situations expérimentales ont été appliquées aux mêmes animaux : un test d'habituation aux conditions d'open-field pendant 7 jours (de 2 jours à 8 jours d'âge), de réaction à un stimulus auditif inhabituel à l'âge de 9 jours, de réaction à un stimulus visuel inhabituel à l'âge de ro jours, et une mesure d'immobilité tonique à l'âge de ir jours.

L'ensemble des résultats des quatre tests est concordant et montre que les animaux actifs en open-field à l'âge de deux jours sont les moins émotifs. Les animaux émotifs sont caractérisés en open-field par une latence longue, une faible activité et des piaulements, défécations et sauts peu fréquents. On doit remarquer en particulier la relation entre déjections et émotivité qui est l'inverse de celle observée chez les rongeurs, et celle observée entre piaulements et émotivité qui renforce les réserves déjà émises par d'autres auteurs quant aux appellations telles que " distress call " utilisées pour désigner le piaulement.

L'effet principal de l'émotivité semble bien être ur effet inhibiteur portant aussi bien sur l'activité que sur les sauts, les cris ou les déjections.
\end{abstract}

\section{INTRODUCTION}

L'étude du comportement en open-field pose le problème de la liaison entre les comportements observés et l'émotivité de l'animal. Ce problème posé par HALL (I934) a souvent été discuté depuis chez les rongeurs de laboratoire (SNOwDON, BELI, Henderson, I964; Ader, r969; Archer, r973). Chez le poussin, les études ont surtout porté sur le rôle des conditions d'élevage ou de testage sur la réaction d'immobilité tonique unanimement considérée comme une bonne mesure d'émotivité (Salzen, I963; Candland, Nagy, Conklyn, I963; Gallup et al., I970, I972; GALLUP, I973). 
D'autre part, il a été montré (BOYER et al., I973; FAURE et FolMER, I975), que 1'activité en open-field est un critère de mesure facilement utilisable pour une expérience de sélection sur le comportement. Nous avons donc tenté de relier les différentes mesures prises en open-field avec d'autres mesures d'émotivité.

\section{I. - MATÉRIEL ET MÉTHODES}

\section{I. - Animaux}

Les animaux utilisés proviennent d'une souche de type Cornish sélectionnée de manière divergente sur l'activité en open-field mesurée à l'âge de deux jours (FAURE et Folmer, I975.

Ces animaux proviennent de la deuxième génération de sélection. Pendant toute la période d'expérimentation, les animaux des deux souches ont été élevés séparément. Soixante-dix-huit poussins provenant de la souche "actifs" (A) et 80 poussins de la souche "inactifs "(I) ont été utilisés.

Les sexes ne seront pas séparés pour le dépouillement, car nous n'avons observé aucune différence significative entre mâles et femelles.

\section{2. -- Méthode expérimentale}

\section{- Comportement en open-field.}

Les poussins utilisés ont été testés pour leur comportement en open-field pendant 7 jours à partir de l'âge de 2 jours. Cette expérience doit permettre de mesurer la vitesse à laquelle les anımaux s'habituent à l'open-field, milieu nouveau. C'est donc une mesure d'émotivité. L'openfield utilisé est une cuve circulaire de I mètre de diamètre, équipée de cellules photo-électriques permettant l'enregistrement automatique de la latence et du nombre de faisceaux interrompus (nombre de " tops ") (pour plus de détails, voir Faure et Folmer, 1975. Les animaux disposent pour chaque test de 100 secondes. Si, à la fin de ce temps, le poussin n'a pas interrompu de faisceau, son activité est notée o " top " et sa latence égale à Ioo secondes. S'il interrompt un faisceau lumineux avant la fin des roo secondes la latence est notée et le nombre de "tops " est compté pendant les roo secondes consécutives à la rupture du premier faisceau.

En plus de ces deux critères, sont notés : le nombre de cellules utilisées, le nombre de sauts, les déjections et les cris. La note est o si le poussin émet le gazouillis (twitter, pleasure call, Collias (1952), Collias et Joos (1953), Montevecchi et al. (1973)), ou est silencieux pendant plus de $80 \mathrm{p}$. Ioo du temps, I entre $80 \mathrm{p}$. 100 et $20 \mathrm{p}$. I00, 2 pour moins de $20 \mathrm{p}$. IoO.

\section{- Tests d'immobilité.}

Trois tests d'émotivité ont été appliqués aux mêmes animaux : réaction à un stimulus auditif inhabituel à l'âge de 9 jours, réaction à un stimulus visuel inhabituel à l'âge de ro jours, réaction cl'immobilité tonique à l'âge de I I jours.

Pour les deux stimuli inhabituels, l'épreuve a eu lieu dans l'open-field précédemment utilisé. L'animal prélevé dans son groupe d'élevage est placé dans l'open-field et l'expérimentateur attend que le poussin commence à émettre le piaulement (peep, distress call, Collias (I952), Collias et Joos (1953), Montevecchi et al. (1973), avant de déclencher le stimulus (ceci afin de tester tous les animaux dans un état émotif comparable).

Le stimulus auditif est une fréquence pure de 2 ooo $\mathrm{Hz}$ émise pendant 5 secondes. Le stimulus visuel est constitué de deux disques de $15 \mathrm{~cm}$ de diamètre, extérieurement peints en noir (comme l'open-field) et intérieurement peints de 3 disques concentriques de l'intérieur vers l'extérieur noir, orange et blanc. Au moment de l'introduction du poussin, les deux disques sont plaqués l'un contre l'autre et peuvent s'ouvrir, grâce à un ressort, quand l'expérimentateur le désire.

La réaction d'immobilité tonique a été provoquée en maintenant l'animal en position couchée pendant 5 secondes.

Pour chacun de ces 3 tests, il a été attribué un point par animal chaque fois qu'il reste immobile pendant les deux minutes consécutives à la fin du stimulus ou après qu'il ait été lâché, dans le cas du test d'immobilité tonique. Chaque animal a donc eu une note d'émotivité variant de o à 3 , les animaux notés o étant les moins émotifs. 


\section{II. - RÉSULTATS}

\section{r. - Comportement en open-field}

L'aspect qualitatif de ces comportements a déjà été décrit, ainsi que les différences entre les deux souches utilisées (FAURE et FolmER, I975).

Nous nous sommes surtout attachés à l'étude des relations entre les différentes mesures. Les corrélations des mesures des différents jours sont présentées dans le tableau I pour le produit nombre de "tops " $\times$ nombre de cellules utilisées. Ce produit représente mieux que le nombre de top seul, l'activité des animaux. En effet, un poussin peut marquer un nombre de " tops " élevé en restant devant une seule callule et en picorant par exemple. Il est bien évident qu'un tel animal ne peut être considéré comme actif. Le tableau I montre que pour les "actifs ", les corrélations entre les mesures d'activité des 7 jours sont toujours positives et toutes significatives, sauf une (jour I avec jour 6). Par contre, il permet de constater que pour les " inactifs ", la mesure prise le premier jour n'est pas en corrélation avec les suivantes. Par contre, à partir $\mathrm{du} 2^{\mathrm{e}}$ jour, toutes les mesures sont liées positivement entre elles et de manière significative, sauf entre les jours $2-3$ et $2-4$. Il semble donc que dans le cas des " inactifs " et dans ce cas seulement la mesure prise le premier jour est différente de celles qui sont prises les jours suivants.

\section{TABLEAU I}

Matrice des corrélations entre les mesures d'activité en open-field prises 7 jours consécutifs

L'activité est mesurée par le produit du nombre de "tops" par le nombre de cellules utilisées.

Les valeurs situées au-dessus de la diagonale sont celles obtenues pour les actifs et celles situées au-dessous pour les inactifs

\begin{tabular}{|c|c|c|c|c|c|c|c|}
\hline Jour & 1 & 2 & 3 & 4 & 5 & 6 & 7 \\
\hline 1 & & $\begin{array}{l}0,53 \\
* * *\end{array}$ & $\begin{array}{c}0,25 \\
*\end{array}$ & $\begin{array}{l}0,14 \\
* * *\end{array}$ & $\begin{array}{c}0,31 \\
* *\end{array}$ & 0,15 & $\begin{array}{c}0,28 \\
*\end{array}$ \\
\hline 2 & $-0,05$ & & $\begin{array}{r}0,57 \\
* * * \\
\end{array}$ & $\begin{array}{c}0,70 \\
* * * \\
\end{array}$ & $\begin{array}{c}0,53 \\
* * * \\
\end{array}$ & $\begin{array}{c}0,56 \\
* * *\end{array}$ & $\begin{array}{l}0,47 \\
* * *\end{array}$ \\
\hline 3 & 0,01 & 0,19 & & $\begin{array}{l}0,61 \\
* * *\end{array}$ & $\begin{array}{l}0,53 \\
* * *\end{array}$ & $\begin{array}{l}0,57 \\
* * *\end{array}$ & $\begin{array}{l}0,34 \\
* * *\end{array}$ \\
\hline 4 & $-0,04$ & 0,18 & $\underset{* *}{0,32}$ & & $\begin{array}{c}0,66 \\
* * *\end{array}$ & $\begin{array}{l}0,67 \\
* * *\end{array}$ & $\begin{array}{c}0,62 \\
* * *\end{array}$ \\
\hline 5 & $-0,01$ & $\begin{array}{l}0,44 \\
* * *\end{array}$ & $\begin{array}{c}0,27 \\
*\end{array}$ & $\begin{array}{c}0,27 \\
*\end{array}$ & & $\begin{array}{l}0,69 \\
* * *\end{array}$ & $\begin{array}{c}0,56 \\
* * *\end{array}$ \\
\hline 6 & 0,06 & $\begin{array}{c}0,35 \\
* * \\
\end{array}$ & $\begin{array}{c}0,26 \\
* \\
\end{array}$ & $\begin{array}{c}0,39 \\
* * * \\
\end{array}$ & $\begin{array}{c}0,64 \\
* * *\end{array}$ & & $\begin{array}{r}0,60 \\
* * *\end{array}$ \\
\hline 7 & 0,00 & $\begin{array}{c}0,28 \\
*\end{array}$ & $\begin{array}{l}0,42 \\
* * *\end{array}$ & $\begin{array}{l}0,48 \\
* * *\end{array}$ & $\begin{array}{l}0,52 \\
* * *\end{array}$ & $\begin{array}{l}0,53 \\
* * *\end{array}$ & \\
\hline
\end{tabular}




\section{TABLEAU 2}

Comparaison des résultats des trois épreuves mettant en évidence l'émotivité par la durée de la réaction d'immobilité consécutive à un stimulus inhabituel

(les valeurs indiquent

le nombre d'animaux se déplaçant dans les deux minutes suivant la stimulation par rapport au nombre d'animaux ayant subi l'épreuve)

\begin{tabular}{|c|c|c|c|}
\hline \multirow{2}{*}{$\begin{array}{l}\text { Conditions } \\
\text { de mise en évidence } \\
\text { de l'émotivité }\end{array}$} & \multicolumn{3}{|c|}{ Souche } \\
\hline & Actifs & Inactifs & $\mathbf{P}(\mathbf{1})$ \\
\hline Stimulus audif & $40 / 76$ & $14 / 69$ & $1 \%$ \\
\hline Stimulus visuel & $60 / 73$ & $42 / 61$ & $10 \%$ \\
\hline Immobilité tonique & $20 / 76$ & $10 / 72$ & $5 \%$ \\
\hline
\end{tabular}

(1) Test du $\chi^{2}$.

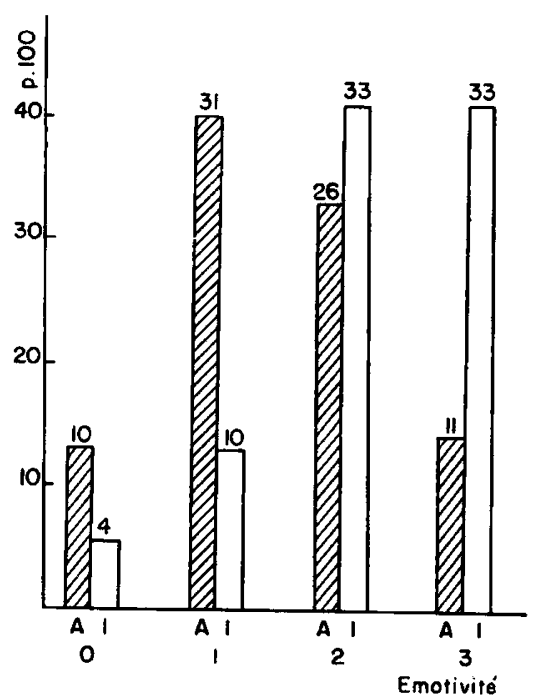

FIG. x. - Répartition des notes d'émotivité

(nombre de tests où l'animal reste immobile pendant 2 minutes)

"Les chiffres indiqués au-dessus de l'histogramme représentent le nombre d'animaux dans chaque classe

En grisé, les actifs; en blanc, les inactifs 
Ceci montre que les " actifs " s'adaptent à la situation expérimentale dès le premier passage alors que l'adaptation n'apparaît qu'à partir du $2^{\mathrm{e}}$ passage chez les " inactifs ».

\section{2. - Tests d'immobilité}

Le nombre d'animaux dont l'immobilité n'a pas dépassé 2 minutes après la stimulation diffère en fonction de la souche dans chacune des trois épreuves (tabl. 2 et fig. I) ce sont les inactifs qui présentent les réactions d'immobilité les plus stables. Ce résultat nous conduit donc à conclure que les poussins " inactifs " sont les plus émotifs.

\section{3. - Relations entre émotivité et comportement en open-field}

L'activité moyenne en open-field, calculée sur les 7 jours de mesure apparaît en relation avec les trois mesures spécifiques d'émotivité (fig. 2). Ceci confirme l'hypothèse selon laquelle les animaux les plus émotifs sont aussi les moins actifs.

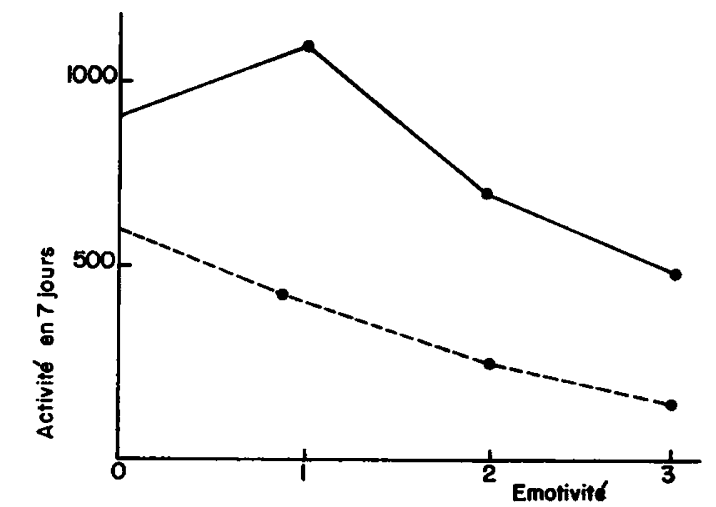

FIG. 2. - Relation entre l'activité en open-field et l'émotivité (traits pleins : actifs; tirets : inactifs)

De même on peut remarquer que la latence moyenne de départ en open-field est d'autant plus longue que le poussin est plus émotif et ceci dans les deux souches (fig. 3).

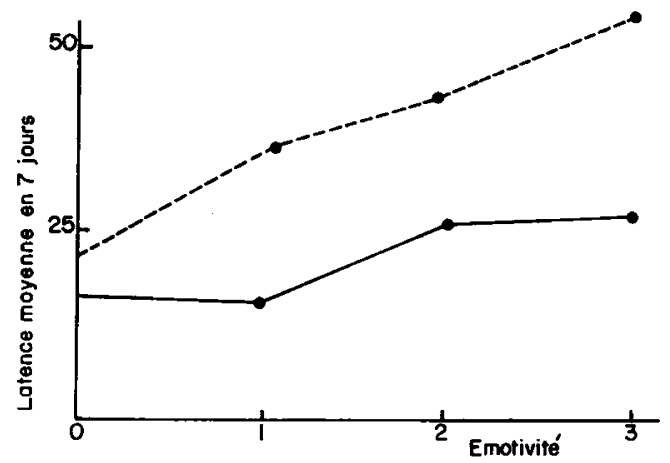

FIG. 3. - Relation entre la latence du premier déplacement en open-field et l'émotivité (traits pleins : actifs; tirets : inactifs) 
Les émissions sonores, les réactions de saut et les défécations sont également en relation avec les mesures d'émotivité : la figure 4 montre que les animaux les moins émotifs sont aussi ceux qui défèquent le plus et qui émettent le plus de piaulements, aussi bien dans la souche d' " actifs " que dans la souche d' "inactifs ", et que les moins émotifs sont aussi ceux qui effectuent le plus de sauts (la courbe n'a été figurée que pour les A, car pour les I, seuls 5 animaux sur 80 ont sauté au cours des 7 passages en open-field).

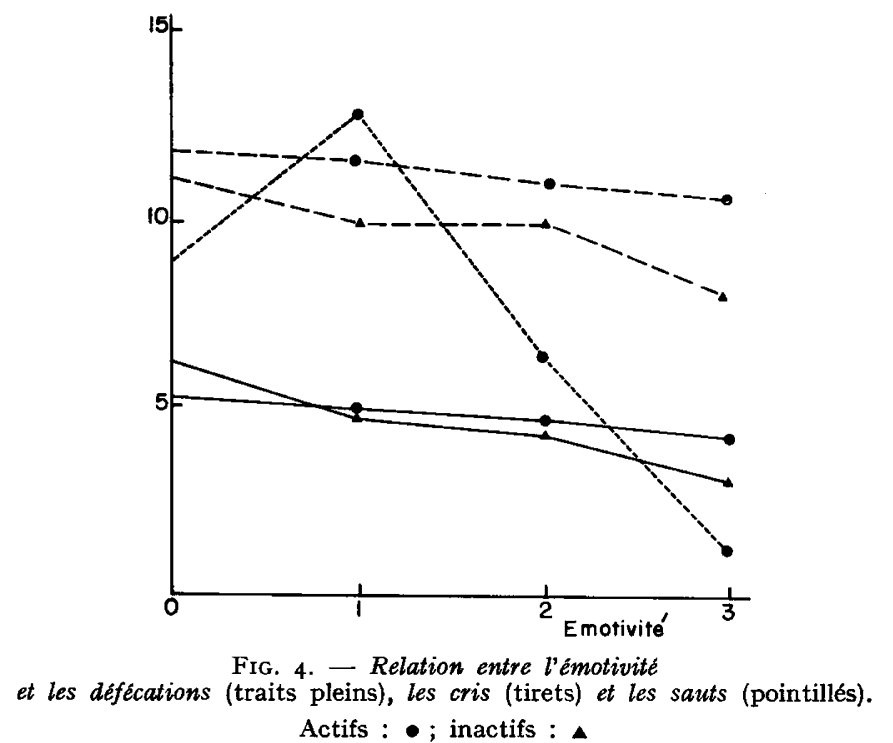

III. DISCUSSION

La souche d'actifs est caractérisée par une ambulation importante, une latence courte, beaucoup de déjections, de piaulements et de sauts. Les épreuves d'émotivité démontrent d'autre part que les " actifs" sont moins émotifs que les "inactifs ". De plus, les figures 2,3 et 4 ainsi que le tableau 3 qui récapitule à l'intérieur de chacune des deux souches, les différences entre animaux classés émotifs (notes 2 et 3 ) et non émotifs (notes o et I) montrent que dans les deux souches, les animaux émotifs sont les moins actifs, ceux qui présentent la latence la plus longue, qui émettent le moins de piaulements, qui défèquent et qui sautent le moins.

Ces résultats montrent que l'activité en open-field à deux jours est une mesure utilisable de l'émotivité, puisque dès $\mathrm{la}_{2}$ les deux souches présentent des différences importantes pour ce caractère. Chez le jeune poussin, l'animal non émotif est donc celui qui émet le plus de piaulements. Ceci confirme donc les résultats de MonThevecchi et al. (I973), et renforce les réserves qu'ont émises ces auteurs sur l'utilisation d'appellations telles que distress call (CoLLIAS et Joos, I953), pour désigner cette émission sonore. 
D'autre part, ce sont les moins émotifs qui défèquent le plus. Ces résultats sont en contradiction avec tous les résultats obtenus chez les rongeurs, mais sont en accord avec les résultats de BouRdens (I97r) chez le Faisan. Il semble donc que chez les gallinacés les déjections ne présentent pas la même relation avec l'émotivité que celle décrite chez les rongeurs. Cependant, il faut noter que CANDLAND et al. (1963) trouvent une corrélation positive entre les défécations et les réactions d'immobilité chez le poussin. Les résultats peuvent donc dépendre des souches utilisées (CANDLAND et al., I963, utilisent une souche Leghorn blanche, alors que nous utilisons une souche Cornish) ou des conditions d'élevage ou de testage des animaux. Il est donc difficile de généraliser. Il faut cependant noter que chez les poussins (au moins dans le cas de notre souche) et chez le faisandeau, les déjections et l'émotivité présentent une relation inverse de celle observée chez les rongeurs.

\title{
TABLEAU 3
}

\begin{abstract}
Différences entre émotifs (notes 2 et 3 )
et non émotifs (notes o et I) dans les deux souches étudiées pour les différents caractères mesurés en 7 tests d'open-field
\end{abstract}

\begin{tabular}{|c|c|c|c|c|c|c|}
\hline & \multicolumn{3}{|c|}{ Actifs } & \multicolumn{3}{|c|}{ Inactifs } \\
\hline & Émotifs & $\begin{array}{c}\text { Non } \\
\text { émotifs }\end{array}$ & $\mathbf{P}$ & Émotifs & $\begin{array}{c}\text { Non } \\
\text { émotifs }\end{array}$ & $\mathbf{P}$ \\
\hline Activité (1) & 649 & 1066 & $1 \%$ & 201 & 477 & $1 \%$ \\
\hline Latence $(\mathbf{l})$ & 186 & 110 & $1 \%$ & 340 & 216 & $1 \%$ \\
\hline Déjections $\left({ }^{(2)}\right.$ & 4,56 & 5,00 & NS & 3,73 & 5,21 & $1 \%$ \\
\hline Cris $\left({ }^{2}\right)$ & 13,00 & 13,80 & $1 \%$ & 10,56 & 12,14 & $5 \%$ \\
\hline Sauts ( $\left.{ }^{8}\right)$ & 4,84 & 11,93 & $1 \%$ & & & \\
\hline
\end{tabular}

(1) Test $t$ de Student.

(2) Test U de Mann et Whitney (SIEgeL, 1956).

Ces résultats montrant que les " actifs " sont les moins émotifs permettent d'expliquer les valeurs obtenues pour les corrélations entre les différentes mesures d'activité. On peut en effet penser que chez les inactifs le premier jour on mesure la réaction à un milieu nouveau, alors que dès le deuxième jour, il $\mathrm{y}$ a habituation et que la mesure n'est plus qu'une mesure d'activité motrice. Pour les actifs, peu émotifs, 1'habituation serait immédiate et la mesure ne représenterait que l'activité motrice. Cette hypothèse n'explique cependant pas que les inactifs ne rattrapent jamais le niveau d'activité de la souche d'actifs.

Il semble bien que l'effet le plus marqué de l'émotivité est un effet inhibiteur agissant aussi bien sur l'activité que sur les déjections, les cris ou les sauts.

Ces résultats nous paraissent aussi importants du point de vue pratique puisque 
la méthode de sélection en open-field est suffisamment rapide pour être appliquée à des souches de dimension commerciale et que ces résultats montrent qu'elle permet de sélectionner les animaux sur leur émotivité.

Reçu pour publication en mars 1975.

\section{SUMMARY}

\section{STUDY ON CONNECTION BETWEEN BEHAVIOR IN OPEN-FIELD} AND EMOTIVITY IN YOUNG CHICKEN

We have studied connection between activity in open-field and emotivity in two strains of chicks selected for their activity in open-field when two days old. The same birds went through four experimental situations : one test to become habituated to open-field conditions during seven days (from 2 to 8 days of age), one test of reaction to unusual auditory stimulus when 9 days old, one test of reaction to an unusual visual stimulus at Io days of age and one measurement of tonic immobility at I days of age.

The results of the four tests, as a whole, are agreeing and show that active birds at 2 days of age in open-field are the least emotive. Emotive birds are caracterized in open-field by a long latency, a low activity, few peepings, few jumps and defecations. It is to be pointed out relation between dejecta and emotivity which is the opposite of the one observed in the case of rodents, and relation observed between peepings and emotivity which strengthens reserves already made by other authors as for ways of naming like " distress call " used to design peeping.

The main effect of emotivity seems to be an inhibitory effect bearing on activity as well as on jump, calls or dejecta.

\section{RÉFÉRENCES BIBLIOGRAPHIQUES}

Ader R., I969. Adrenocortical function and the mesurement of emotionality. III. Open-fiels behavior. Ann. N. Y. Acad. Sci., 159, 79I-805.

Archer J., 1973. Tests for emotionality in rats and mice : a review. Anim. Behav., 21, 205-235.

Bourdens P., I97I. Le Faisan de repeuplement. Une approche par la génétique du comportement. Essai de sélection par des tests précoces. Premiers résultats. Mémoire de fin d'études E. S. A., Purpan-Toulouse.

Boyer J. P., MELIN J. M., Bourdens P., 1973. Behavioral genetics. Results of an activity-inactivity test in young pheasants. Ann. Genet. Sel. Anim., 5, 417-418.

Candland D. K., Nagy Z. M., Conklyn D. H., 1963. Emotional behavior in the domestic chicken (White Leghorns) as a function of age and developmental environment. J. Comp. Physiol. Psychol., 56, ro69-I073.

Collias N. E., 1952. The development of social behavior in birds, $A u k, 69$, I27-I59.

Collias N. E., Joos M., I953. The spectrographic analysis of signals of the domestic fowl. Behaviour, 5, I76-I88.

Faure J. M., Folmer J. C., I975. Étude génétique de l'activité précoce en open-field du jeune poussin. Ann. Génét. Sél. Anim., 7, 123-132.

GalluP G. G., 1973. Tonic immobility in chickens : is a stimulus that signals shock more aversive than the receipt of shock? Anim. Behav., 1, 228-232.

Gallup G. G., Nash R. F., Potter R. J., Donegan N. H., I97o. Effect of variying conditions of fear on immobility reactions in domestic chickens (Gallus gallus). J. Comp. Physiol. Psychol., 73, 442-445.

Gallup G. G., Rosen T. S., Brown C. W., I972. Effect of conditioned fear on tonic immobility in domestic chickens. J. Comp. Physiol. Psychol., 78, 22-25.

Hall C. S., I934. Emotional behaviour in the Rat. I. Defecation and urination as mesures of individual differences in emotionality. J. Comp. Psychol., 18, 385-403.

Montevecchi W. A., Gallup G. G., Dunlap W. P., I973. The peep vocalization in group reared chicks (Gallus domesticus) : its relation to fear. Anim. Behav., 21, 116-123.

SAlzen E. A., I963. Imprinting and the immobility reactions of domestic fowl. Anim. behat), 11, 66-71.

Siegel S., I956. Non parametric statistics for the behavioral science, Mac Graw-Hill, New-York.

Snowdon C. T., Bell D. D., Henderson N. D., I964. Relationships between heart rate and openfield behavior. J. Comp. Physiol. Phychol., 58, 423-426. 